\title{
Phenytoin-induced gingival overgrowth associated with generalized periodontitis- a case report.
}

\author{
Jithendra K. D. Proff \& Head
Department of Periodontics, Kanti Devi Dental College and Hospital, \\ National Highway \# 2, Chattikara Post, 281006,Mathura, Uttar Pradesh
}

Case Presentation: A 45 year old male patient reported to the Department of Periodontics with a chief complaint of swollen and bleeding gums since 2 years (Figure 1). Patient's history stated that initially the overgrowth was minimal, however with the passage of time gingival overgrowth involved more areas of the gingiva and became more pronounced. Patient's oral hygiene status was poor with generalized exudation and pus discharge. Halitosis was also present. Periodontal findings included generalized deep periodontal pockets and periodontal abscess in the mandibular left first molar region. Overgrowth of the gingiva was diffuse, generalized and combined (having both inflammatory and fibrotic components) in nature. Overgrowth was painless, beadlike, pronounced in the maxillary and mandibular anterior region. Gingival overgrowth was graded as class ?? according to Bokenkamp et al.1 Orthopantomograph showed generalized interproximal bone loss (Figure 4). Generalized mobility of the teeth was noted. Further, clinical examination revealed that patient had his maxillary left central incisor extracted due to periodontal problems around two years back. Patient was suffering from grand mal epilepsy and was on phenytoin sodium since 15 years. Complete hemogram revealed a normal blood picture and random blood sugar estimation ruled out any increased glycemic levels. A diagnosis of drug induced gingival overgrowth with chronic generalized periodontitis was made.

Maxillary right central incisor was extracted as it was considered unsalvageable. Patient was explained regarding the problem and oral hygiene instructions were given. Initially, full-mouth scaling and root planning, antibiotic therapy (Azithromycin $500 \mathrm{mg}$ stat on first day, $250 \mathrm{mg}$ per day for next four days) and phenytoin withdrawal (replacement with valproic acid) were performed; however, clinical results demonstrated partial resolution of gingival overgrowth.

Internal bevel gingivectomy was performed to remove the overgrown gingiva; an internal bevel incision (First incision) was placed with a No. 15 B.P.blade followed by crevicular incisions (Second incision) with a No. 12. B.P.blade. Interdental incisions (Third incision) were placed and a wedge of overgrown gingiva was removed. Mucoperiosteal flaps were elevated and degranulation was done. Surfaces of the root were planned and bony defects were debrided. Inner surface of the mucoperiosteal flaps were thinned with soft tissue nippers. Flaps were replaced back to their original position and sutured. The wedge of overgrown gingiva was sent for histopathologic examination. Removable prosthesis was fabricated for the missing maxillary central incisors. Gingival enlargement did not recur and probing depths had stabilized during the six month recall period Histopathologic examination showed pronounced hyperplasia of the connective tissue and epithelium. Epithelium was acanthotic and connective tissue shows densely arranged collagen Bundles with increase in the number of fibroblasts .

Discussion: Drug induced gingival overgrowth is a common side effect of phenytoin administration. Various theories have been put forward to explain the occurrence of gingival overgrowth in phenytoin users. One theory claims that phenytoin has been associated with drug induced gingival overgrowth in the oral cavity probably due to folic acid deficiency. ${ }^{2}$ Folic acid is presented as polyglutamate in foods; it is then converted into monoglutamates by intestinal conjugase. Phenytoin acts by inhibiting this enzyme therefore causing folic acid deficiency. ${ }^{2}$ Another theory proposed by Hassell et al claims that in non-inflamed gingiva, fibroblasts are less active, whereas in inflamed gingiva, fibroblasts are in an active state due to release of inflammatory mediators and growth factors. ${ }^{3}$ These active fibroblasts respond to circulating phenytoin causing overgrowth. Hassell et al stress the fact that high levels of dental plaque and calculus are critical co-factors for the development and severity of 1 phenytoin-induced gingival overgrowth. ${ }^{3}$ Role of genetics in the occurrence of gingival overgrowths in individuals on phenytoin has also been discussed. ${ }^{4}$

These overgrowths usually have two components: fibrotic component and inflammatory component. Gingival overgrowth is initially small and fibrotic in nature; however when the overgrowth increases in size, it acts as an interference to regular plaque removal and oral hygiene maintenance. This results in accumulation of plaque and calculus with subsequent inflammation leading to combined gingival overgrowth. The most effective treatment for drug-induced gingival overgrowth is the withdrawal or substitution of the drug. 
Antiepileptic drugs like valproic acid and carbamezepine are suitable substitutes for phenytoin. These drugs are effective anti-epileptic drugs without accompanying gingival overgrowth. Preferred antibiotic in treating cases of gingival overgrowth with periodontitis is azithromycin. Azithromycin is chosen because its concentration in periodontal lesions is significantly higher than that of normal gingiva. It has been proposed that azithromycin penetrates fibroblasts and phagocytes in concentrations 100 to 200 times greater than that of extracellular compartment. Drug is also actively transported to sites of inflammation by phagocytes and then released directly into sites of inflammation by phagocytes and then released directly into the sites of inflammation as the phagocytes rupture during phagocytosis. ${ }^{5}$

Inflammatory component of overgrowth resolves when the causative local factors (plaque and calculus) are removed. However the fibrotic component has to be excised. Excision of the overgrown gingiva can be performed in one of the two ways; ${ }^{1}$ ) external bevel gingivectomy or conventional gingivectomy and 2) internal bevel gingivectomy or reverse bevel gingivectomy depending on the presence of loss of attachment and amount of width of attached gingiva. ${ }^{5} \mathrm{~A}$ treatment algorithm for persistent overgrowths after Maintenance phase is very vital after comprehensive periodontal therapy is completed. Recurrences of gingival overgrowth which are very common, can be prevented by regular and meticulous plaque control by the patient.

Conclusion: Drug-induced gingival overgrowth remains a significant problem for the clinician. Both clinician and patient have an important role to play in improving oral hygiene and gingival health. This opportunity should be used to establish a close liaison between the patient's physician and the periodontist to try and identify alternative drug regimens that can help reduce the impact of this unwanted effect. Usage of drugs like phenytoin, cyclosporine and calcium channel blockers is going to increase. Thus, the problem of drug-induced gingival overgrowth is also going to increase. This highlights the need to identify the patient at risk from this unwanted effect and developing the appropriate management strategies.

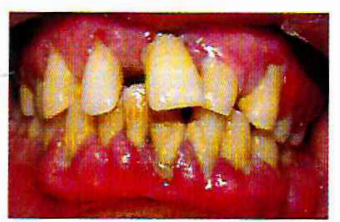

Fig:1

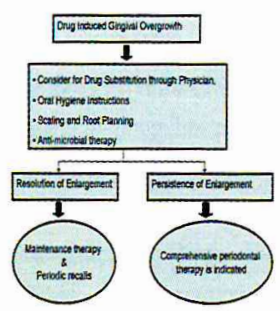

Fig:5

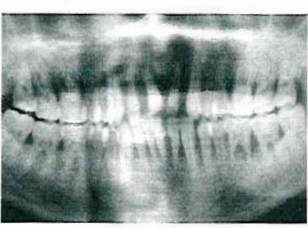

Fig:2

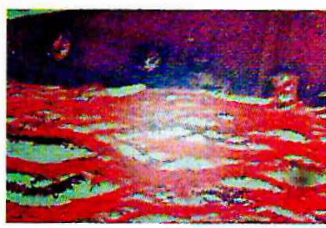

Fig:4

References:

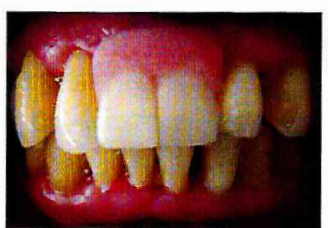

Fig:3

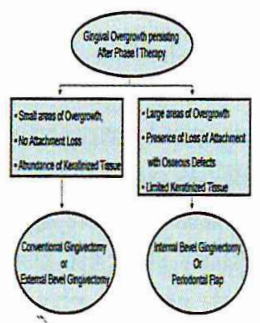

Fig:6

1. Bökenkamp A, Bohnhorst B, Beier C, Albers N, Offner G, Brodehl J. Nifedipine aggravates cyclosporine A-induced gingival hyperplasia. Pediatric Nephrology 1994; 8: 181-185.

2. Prasad VN, Chawla HS, Goyal A, Gauba K, Singhi P. Folic acid and phenytoin induced gingival overgrowth--is there a preventive effect.J Indian Soc Pedod Prev Dent. 2004; 22(2):82-91.

\section{Hassell TM. Gingival overgrowth: hereditary considerations. Compend Suppl. 1990;(14):511-514.}

4. Hassell TM, Burtner AP, McNeal D, Smith RG. Oral problems and genetic aspects of individuals with epilepsy. Periodontol 2000.1994;6:68-78.

5. Namazi $S$, Haghighi $A B$, Karimzadeh I. Is oral azithromycin effective for the treatment of phenytoin-induced gingival overgrowth in epileptic patients? Med Hypotheses. 2007;69(5):1159-1160. 